\title{
Probiotic therapy for the prevention and treatment of Clostridium difficile-associated diarrhea: a systematic review
}

\author{
Nandini Dendukuri, Vania Costa, Maurice McGregor, James M. Brophy
}

Abstract

Background: The recent increase in the number and severity of cases of nosocomial Clostridium difficile-associated diarrhea (CDAD) has prompted interest in the use of probiotics for the prevention and treatment of this disease. We performed a systematic review of randomized controlled trials to assess the effectiveness of probiotic therapy.

Methods: We searched the PubMed, EMBASE, INAHTA, HEN and Cochrane Collaboration databases to identify trials in which the prevention or treatment of CDAD with probiotic therapy was the primary or secondary outcome. We extracted data on the number of patients randomly assigned to receive probiotic or placebo, the number of patients with CDAD, the type of probiotic, criteria for diagnosing CDAD, persistence of infection after treatment, compliance and adverse effects.

Results: We identified 4 eligible studies in which prevention $(n=1)$ or treatment $(n=3)$ of CDAD was the primary outcome. The benefit of probiotic therapy seen in 2 of the studies was restricted to subgroups characterized by severe CDAD and increased use of vancomycin. The remaining 2 studies were too methodologically flawed for us to draw meaningful conclusions. We also identified 4 trials in which prevention of antibiotic-associated diarrhea with probiotics was the primary outcome and prevention of CDAD a secondary outcome. These studies were limited primarily by too few CDAD cases and provided no evidence of effective prophylaxis. Overall, heterogeneity in choice and dose of probiotic and in criteria for diagnosing CDAD makes it difficult to synthesize information from the 8 studies.

Interpretation: Studies conducted to date provide insufficient evidence for the routine clinical use of probiotics to prevent or treat CDAD. Better designed and larger studies are needed.

CMAJ 2005;173(2):167-70

$\mathrm{B}$ oth the incidence and severity of Clostridium difficileassociated diarrhea (CDAD) have increased in hospitals across North America. ${ }^{1-4}$ Recent outbreaks in Montreal and Sherbrooke resulted in a 4-fold increase in the number of CDAD cases as well as an increase in the number of attributable deaths. ${ }^{1,5-7} \mathrm{CDAD}$ is a debilitating and costly illness, particularly among patients with recurring episodes. Probiotics, or naturally occurring "good bacteria," have been suggested as a means of both preventing and treating the disease. ${ }^{8-11}$ The disturbance of normal gastrointestinal flora, particularly after antibiotic use, is be- lieved to predispose patients to colonization by $C$. difficile; ${ }^{2}$ probiotics, by delivering bacteria to the gastrointestinal tract, are believed to restore equilibrium in the altered gastrointestinal flora and thus protect against colonization. ${ }^{8}$ Probiotics that have been proposed for prevention and treatment of antibiotic-associated diarrhea and CDAD include various bacteria (Bifidobacterium, Lactobacillus GG, $L$. rhamnosus, L. casei, L. plantarum 299v, Enteroccus faecium [SF68]) and yeasts (Saccharomyces boulardii, S. cerevisiae). They are commonly available as lyophilized capsules or in the form of a fermented drink. We performed a systematic review of randomized controlled trials of probiotic therapy for the prevention and treatment of CDAD.

\section{Methods}

We followed the guidelines for systematic reviews described in the QUORUM statement. ${ }^{12}$ We searched the databases of PubMed, EMBASE, INAHTA, HEN and the Cochrane Collaboration up to Mar. 19, 2005, using the following search terms: (Probiotic, Probiotics, Lactobacillus, lactic-acid, acidophilus, casei, bulgaricus, plantarum, rhamnosus, yeast, Saccharomyces, boulardii, cervisiae, Bifidobacterium, bifidum, SF68) and (Clostridium, difficile, diarrhea, antibiotic-associated) and (patients, subjects). We selected randomized controlled trials where prevention or treatment of $\mathrm{CDAD}$ was either a primary or secondary outcome and where the study subjects were adults and recruited from a hospital setting. Further details of the database search strategy are given in online Appendix 1 (www.cmaj.ca/cgi/content/full /173/2/167/DC1). We also reviewed the reference lists from articles fulfilling the inclusion criteria.

Two of the authors independently extracted the following data from all studies meeting the inclusion criteria: the number of patients randomly assigned to probiotic and placebo groups, the number of patients with $\mathrm{CDAD}$ in each group, the type of probiotic, criteria for diagnosing $\mathrm{CDAD}$, persistence of $C$. difficile infection after treatment, compliance and adverse effects. We calculated a risk difference and $95 \%$ confidence interval (CI) for comparing probiotic and placebo groups. CIs were calculated using the hybrid score method with continuity correction. ${ }^{13}$

The quality of each trial was assessed using the scale described by Jadad and associates ${ }^{14}$ which rates studies on randomization, blinding and attrition. The score ranges from 0 to 5 , with 5 indicating best quality. A more detailed summary of each study is given in a recent Health Technology Assessment report prepared by the Technology Assessment Unit of the McGill University Health Centre ("Probiotics," available at www.mcgill.ca/tau/publications/2005/). 


\section{Results}

The results of our search strategy are summarized in Fig. 1 . We identified 4 studies in which prevention $(n=1)^{15}$ or treatment $(n=3)^{16-19}$ of CDAD was the primary outcome. No meta-analysis was performed because there was marked heterogeneity between the studies. We also identified 4 randomized, placebo-controlled trials of probiotic therapy for the prevention of antibiotic-associated diarrhea where prevention of CDAD was measured as a secondary outcome ${ }^{19-22}$ (Table 1; a longer version of this table is available online at www.cmaj.ca/cgi/content/full/1723/2/167/DC1). Study quality ranged from 3 to 5 , with all the randomized studies being double-blinded.

As seen in Table 1, there was considerable protocol variation in the choice of probiotic formulation and duration of treatment. There was also variability in the criteria for defining a case of CDAD: various definitions of diarrhea and different combinations of tests for $C$. difficile and its associated toxins A and B were used. In some studies patients were considered to have CDAD if they had diarrhea and were positive for $C$. difficile as determined by at least 1 standard culture or toxin A or B test. This definition, although probably the most inclusive, has a low specificity because it classifies patients with nontoxigenic $C$. difficile as having CDAD. ${ }^{2}$ In other studies, patients were tested for toxin presence only if a standard culture was positive for the bacillus. However, the poor sensitivity of standard culture means that subjects who had a false-negative result may not have been tested.

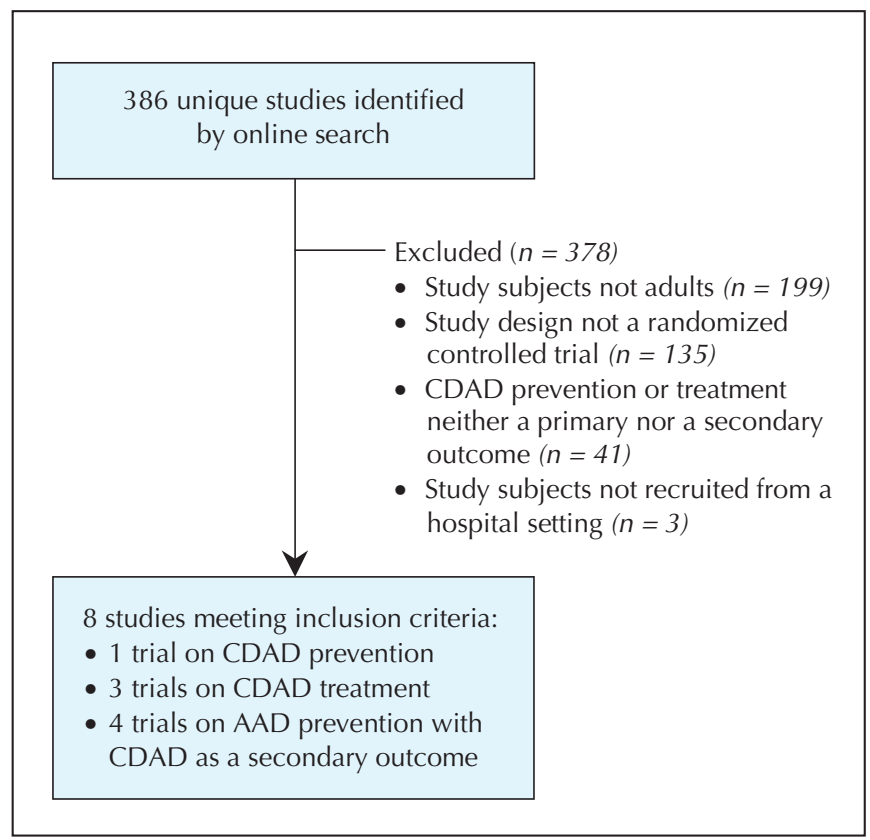

Fig. 1: Flow of articles in the search strategy. CDAD = Clostridium difficile-associated diarrhea, ADD = antibioticassociated diarrhea. No new studies were found when the reference lists of eligible studies were reviewed.
In the single study on the effectiveness of probiotic therapy in preventing CDAD,${ }^{15}$ no difference was found between probiotic and placebo groups in the percentage of patients who tested positive for $C$. difficile infection after treatment (Table 1). The authors also reported a 32\% (statistically nonsignificant) decrease in the incidence of diarrhea among patients who were positive for infection after culture and toxin test, but this comparison is not appropriate since it is not between all subjects randomly assigned to the 2 groups.

Among the 3 trials of probiotic treatment of CDAD, ${ }^{16-19}$ only 1 , by McFarland and associates ${ }^{16}$ reported a significant beneficial effect overall. However, in a post hoc analysis, the authors found that this effect was almost entirely limited to a subgroup with recurrent CDAD (risk difference $30,95 \%$ CI 2.3 to 50.6 ). These subjects were characterized by a greater use of vancomycin, although vancomycin use was not significantly associated with the outcome in a multivariate analysis. In the study by Surawicz and colleagues, ${ }^{17}$ a subgroup analysis of subjects receiving high-dose vancomycin, who were more likely to have severe CDAD, demonstrated a beneficial effect of the probiotic (risk difference $33,95 \% \mathrm{CI}-0.3$ to 62.0 ). However, the CIs in the subgroup analyses from both studies were very wide, which made it difficult to ascertain the magnitude of the effect. The study by Wullt and associates ${ }^{18}$ was chiefly limited by the very small sample $(n=21)$.

In the 4 studies in which prevention of antibiotic-associated diarrhea was the primary outcome, probiotic therapy had no significant effect on the prevention of CDAD. ${ }^{19-22}$ In 1 study, ${ }^{20}$ the only available data on CDAD involved a comparison that was not between randomly assigned subjects. Probiotic therapy was found to be beneficial in preventing antibiotic-associated diarrhea in only 2 of the studies..$^{19,20}$ Within each study there was variability in the antibiotic therapy (type, dose and duration) used concomitantly with the probiotic.

No serious adverse effects or problems with compliance were found in the probiotic group in any of the studies.

\section{Interpretation}

We identified 8 eligible trials of probiotic therapy in which CDAD prevention or treatment was either a primary or secondary outcome. The scant literature does not provide convincing proof of the clinical benefit of probiotics. Interestingly, in 2 treatment studies ${ }^{16,17}$ a beneficial effect (with a wide CI) was observed in a subgroup of patients characterized by more severe disease. In the first study these subjects had had at least 1 previous episode of CDAD in the last year and were more likely to be taking vancomycin than metronidazole. In the second study the patients were taking high doses of vancomycin and were more likely to have colitis. In both studies the apparent beneficial effect of the probiotic was seen only in patients with severe CDAD. The third treatment study ${ }^{18}$ was too small for us to draw any meaningful conclusion. In all 4 studies where $\mathrm{CDAD}$ prevention or treatment was the pri- 
mary outcome, there was no difference between probiotic and placebo groups in the persistence of $C$. difficile after the intervention. ${ }^{15-18}$ Studies in which prevention of CDAD was measured as a secondary outcome had small samples and varied definitions of CDAD. In 3 studies $^{19,21,22}$ the duration of follow-up after antibiotic use was less than 6 weeks, which may be too short for CDAD to develop. ${ }^{23}$

Some authors have suggested that probiotic therapy has not been found to be beneficial because the doses used were too low. ${ }^{22}$ This may be supported by the fact that in some of the studies involving adults in which no benefit was found, the dose used was the same as that used in pediatric studies that reported positive results..$^{24}$ Another concern is that the probiotic preparations may have become nonviable after manufacture. The effectiveness of the probiotic depends on the number of viable bacteria at the time of use. ${ }^{25}$ Several articles have reviewed commercially available probiotic products such as food supplements, yoghurt and fermented

\section{Table 1: Summary of randomized controlled trials where prevention or treatment of Clostridium difficile-associated diarrhea} (CDAD) was the primary or secondary outcome (abridged)*

\begin{tabular}{lccccc}
\hline Trial & $\begin{array}{c}\text { Probiotic and } \\
\text { duration }\end{array}$ & $\begin{array}{c}\text { Outcome measure } \\
\text { (length of follow-up) }\end{array}$ & $\begin{array}{c}\text { Risk difference (95\% Cl) } \\
\text { (placebo-probiotic) }\end{array}$ & $\begin{array}{c}\text { Quality } \\
\text { score }\end{array}$ & Comments \\
\hline Primary outcome & &
\end{tabular}

\section{Primary outcome}

Plummer et Lactobacillus al, 2004; $;^{15}$ acidophilus and

prevention Bifidobacterium bifidum for $20 \mathrm{~d}$

McFarland Saccharomyces Diarrhea and at least 1 et al, 1994; ${ }^{16}$ boulardii for 4 wk positive assay for $C$. treatment

Surawicz et $S$. boulardii for al, 2000; $;^{17} \quad 28 \mathrm{~d}$ treatment

Wullt et al, L. plantarum 2003; $i^{18} \quad 299 v$ for $38 d$ treatment

\section{Secondary outcome} al, $1989^{19}$

\section{S. boulardii} starting within $48 \mathrm{~h}$ of beginning antibiotic until 2 wk post-antibiotic

McFarland et $S$. boulardii al, $1995^{20}$ starting within $72 \mathrm{~h}$ of beginning antibiotic until 3 antibiotic

Lewis et al, $S$. boulardii for $1998^{21}$ duration of antibiotic treatment

Thomas et al, L. GG starting $2001^{22} \quad$ within $24 \mathrm{~h}$ of beginning antibiotic treatment for $14 \mathrm{~d}$

Diarrhea among $C$. difficile by culture or toxin A or B (8wk)

Presence of $C$. difficile $\quad-2.8(-8.7$ to 15.3$)$ $(20 \mathrm{~d})$

20.5 (2.2 to 37.0$)$

First-time CDAD:

4.9 (-17.6 to 26.4$)$

Recurrent CDAD: 30.0 (2.3 to 50.6)

9.8 (-6.7 to 25.6)

Diarrhea and at least 1 positive assay for $C$. difficile by culture or toxin A or B (8wk)

Diarrhea (5-10 d)

Positive assay for $C$. difficile toxins $\mathrm{A}$ or $\mathrm{B}$ (11-13 d)

Acquired C. difficile after enrolment (duration of probiotic treatment; minimum of $8 \mathrm{~d}$ ) difficile- or toxinpositive subjects (7 wk after probiotic was discontinued) Positive assay for $C$. difficile toxin (duration of antibiotic treatment)

Diarrhea and positive assay for $C$. difficile toxin $(21 \mathrm{~d})$ 33.0 (-0.3 to 62.0) -6.4 (-16.2 to 28.1)

Metronidazole:

$-8.3(-40.3$ to 29.6$)$

$-1.4(-3.5$ to 4.1$)$

$-6.8(-22.6$ to 8.0$)$

$0.7(-3.0$ to 4.5$)$
High-dose vancomycin:

Low-dose vancomycin:

-1.9 (-25.8 to 29.2$)$

-19.4 (-64.7 to 15.5$)$

$-13.3(-25.7$ to 4.7$)$
5

Probiotic resulted in a significant reduction in risk of AAD; $C$. difficile tested only in those with $\geq 3$ stool samples; short follow-up

3 No difference in toxin positivity between treatment arms among patients with positive culture; lower incidence of diarrhea reported among culture- and toxin-positive subjects in probiotic group; short follow-up

5 Concomitant use of different antibiotics for differing lengths of time; no difference in culture positivity between treatment arms, but significantly fewer toxin B positive cases in probiotic group

3 Concomitant antibiotic assigned to patients after randomization; no difference in culture or toxin positivity in subgroup taking high-dose vancomycin; results not reported for other groups

4 Small sample; no difference in culture positivity after treatment among 20 patients who had no diarrhea after 5-10 d

4 Probiotic resulted in a significant reduction in risk of AAD; the only available result for CDAD was not based on a comparison between randomized subjects

$4 \quad$ Probiotic had no effect on AAD; $C$. difficile tested regularly every $4 \mathrm{~d}$ and whenever subjects had diarrhea; small number of CDAD cases; short follow-up

$4 \quad$ Probiotic had no effect on AAD; $C$. difficile toxin results obtained from medical chart; small number of CDAD cases; short follow-up

Note: $\mathrm{Cl}=$ confidence interval, $\mathrm{AAD}=$ antibiotic-associated diarrhea

*See the unabridged table at www.cmaj.ca/cgi/content/full/173/2/167/DC1.

$\dagger$ The quality of the studies was assessed using the scale described by Jadad et al. ${ }^{14} \mathrm{~A}$ score of 5 indicates best quality. 
milk $^{25,26}$ and found that the number of colony-forming units can be much lower than advertised and that the bacterial species listed on product labels are often incorrect. It is also possible that, in prevention trials, the probiotic does not survive to colonize the colon because it is eliminated by the acidity of the stomach or because of concomitant use of antibiotics. Only 1 of the studies we reviewed evaluated the presence of the probiotic in fecal samples. ${ }^{18}$ Studies are needed to establish the minimum effective adult dose. Although side effects appear to be rare, there has been a small number of case reports of fungemia and bacteremia attributed to probiotic use, particularly among immunocompromised patients. ${ }^{27}$ These specific side effects were either not observed or not reported in the studies we reviewed. The varied definitions of $\mathrm{CDAD}$ also complicate the interpretation of these studies.

The studies conducted to date provide insufficient evidence for the routine clinical use of probiotics to prevent or treat CDAD in adults. Better designed trials are therefore needed. To demonstrate a prevention effect, studies would need to randomize a sufficiently large number of patients within categories of antibiotic regimens and have a sufficiently long follow-up after antibiotic use. Therapeutic studies would need to randomize patients within strata defined by the concomitant antibiotic regimen. In both types of studies a clearer rationale is needed for the type and dose of probiotic and for how CDAD is defined. Measuring the levels of probiotic bacteria in the stool of patients given different doses may help to determine acceptable dosing. The need for more rigorous proof of the efficacy of probiotics is particularly important given the increasing problem of nosocomial CDAD in Canada and the simultaneous growth in sales of over-the-counter probiotic products.

This article has been peer reviewed.

From the Technology Assessment Unit, McGill University Health Centre (Dendukuri, Costa, McGregor, Brophy); the Department of Medicine (McGregor, Brophy); and Department of Epidemiology and Biostatistics, McGill University, Montréal, Que. (Dendukuri)

Competing interests: None declared.

Contributors: Nandini Dendukuri, Vania Costa and Maurice McGregor were responsible for the literature searches and data extraction. Nandini Dendukuri, Maurice McGregor and James Brophy were responsible for interpreting the data and drafting the paper. Vania Costa was responsible for revising the paper. All of the authors approved the final version.

\section{References}

1. Eggertson L. C. difficile hits Sherbrooke, Que., hospital: 100 deaths. CMAf 2004;171(5):436.

2. Poutanen SM, Simor AE. Clostridium difficile-associated diarrhea in adults. CMA7 2004;171(1):51-8.
3. Pepin J, Valiquette L, Alary ME, Villemure P, Pelletier A, Forget K, et al. Clostridium difficile-associated diarrhea in a region of Quebec from 1991 to 2003: a changing pattern of disease severity. CMA7 2004;171(5):466-72.

4. Archibald LK, Banerjee SN, Jarvis WR. Secular trends in hospital-acquired Clostridium difficile disease in the United States, 1987-2001. F Infect Dis 2004; 189:1585-9.

5. Valiquette L, Low DE, Pepin J, McGeer A. Clostridium difficile infection in hospitals: a brewing storm. CMA7 2004;171(1):27-9.

6. Loo VG, Libman MD, Miller MA, Bourgault AM, Frenette CH, Kelly M, et al. Clostridium difficile: a formidable foe. CMA7 2004;171(1):47-8.

7. Eggertson L, Sibbald B. Hospitals battling outbreaks of C. difficile. CMA7 2004;171(1):19-21.

8. Sullivan A, Nord CE. Probiotics in human infections. 7 Antimicrob Chemother 2002;50:625-7.

9. Gill HS. Probiotics to enhance anti-infective defences in the gastrointestinal tract. Best Pract Res Clin Gastroenterol 2003;17:755-73.

10. Elmer GW. Probiotics: living drugs. Am 7 Health Syst Pharm 2001;58:1101-9.

11. Johnston BC, Vohra S. Treating C. difficile. CMA7 2005;172(4):447-8.

12. Moher D, Cook DJ, Eastwood S, Olkin I, Rennie D, Stroup DF. Improving the quality of reports of meta-analyses of randomised controlled trials: the QUOROM statement. Quality of reporting of meta-analyses. Lancet 1999; 354:1896-900.

13. Newcombe RG. Interval estimation for the difference between independent proportions: comparison of eleven methods. Stat Med 1998;17:873-90.

14. Jadad AR, Moore RA, Carroll D, Jenkinson C, Reynolds DJ, Gavaghan DJ, et al. Assessing the quality of reports of randomized clinical trials: is blinding necessary? Control Clin Trials 1996;17:1-12.

15. Plummer S, Weaver MA, Harris JC, Dee P, Hunter J. Clostridium difficile pilot study: effects of probiotic supplementation on the incidence of $C$. difficile diarrhoea. Int Microbiol 2004;7:59-62.

16. McFarland LV, Surawicz CM, Greenberg RN, Fekety R, Elmer GW, Moyer $\mathrm{KA}$, et al. A randomized placebo-controlled trial of Saccharomyces boulardii in combination with standard antibiotics for Clostridium difficile disease. $7 A M A$ 1994:271:1913-8.

17. Surawicz CM, McFarland LV, Greenberg RN, Rubin M, Fekety R, Mulligan $\mathrm{ME}$, et al. The search for a better treatment for recurrent Clostridium difficile disease: use of high-dose vancomycin combined with Saccharomyces boulardii. Clin Infect Dis 2000;31:1012-7.

18. Wullt M, Hagslatt ML, Odenholt I. Lactobacillus plantarum 299v for the treatment of recurrent Clostridium difficile-associated diarrhoea: a double-blind, placebo-controlled trial. Scand 7 Infect Dis 2003;35:365-7.

19. Surawicz CM, Elmer GW, Speelman P, McFarland LV, Chinn J, van Belle G. Prevention of antibiotic-associated diarrhea by Saccharomyces boulardii: a prospective study. Gastroenterology 1989;96:981-8.

20. McFarland LV, Surawicz CM, Greenberg RN, Elmer GW, Moyer KA Melcher SA, et al. Prevention of beta-lactam-associated diarrhea by Saccharomyces boulardii compared with placebo. Am 7 Gastroenterol 1995;90:439-48.

21. Lewis SJ, Potts LF, Barry RE. The lack of therapeutic effect of Saccharomyces boulardii in the prevention of antibiotic-related diarrhoea in elderly patients. $\mathcal{F}$ Infect 1998;36:171-4.

22. Thomas MR, Litin SC, Osmon DR, Corr AP, Weaver AL, Lohse CM. Lack of effect of Lactobacillus $G G$ on antibiotic-associated diarrhea: a randomized, placebo-controlled trial. Mayo Clin Proc 2001;76:883-9.

23. Mylonakis E, Ryan ET, Calderwood SB. Clostridium difficile-associated diarrhea: A review. Arch Intern Med 2001;161:525-33.

24. Vanderhoof JA, Whitney DB, Antonson DL, Hanner TL, Lupo JV, Young RJ. Lactobacillus $G G$ in the prevention of antibiotic-associated diarrhea in children. 7 Pediatr 1999;135:564-8.

25. Coeuret V, Gueguen M, Vernoux JP. Numbers and strains of lactobacilli in some probiotic products. Int 7 Food Microbiol 2004;97:147-56.

26. Huff BA. Caveat emptor: "Probiotics" might not be what they seem. Can Fam Physician 2004;50:583-7.

27. Marteau P, Seksik P. Tolerance of probiotics and prebiotics. 7 Clin Gastroenterol 2004;38(6 Suppl):S67-9.

Correspondence to: Dr. Nandini Dendukuri, Technology Assessment Unit, R4.09 Ross Pavilion, Royal Victoria Hospital, Montréal QC H3A 1A1; fax 514 843-1493;

nandini.dendukuri@mcgill.ca 